\title{
Digital Transformation in HR
}

\author{
Jakub Chán ${ }^{1, *}$, Miluše Balková ${ }^{1}$ \\ ${ }^{1}$ Institute of Technology and Business, Faculty of Corporate Strategy, Okruzni 517/10, 37001 Ceske Budejovice, Czech Republic
}

\begin{abstract}
The onset of the fourth industrial revolution and lack of human capital puts pressure on the development of own staff in terms of improving their qualifications. In the last four years, there has thus been introduced new methods in the management of staff development. The paper builds on the available texts in the area of theory and digital transformation of talent management and recruiting and puts them into the context of requirements arising from the principles of the fourth industrial revolution. Using matrix analysis, it examines the degree of compliance with the available implementation possibilities. The analysis performed leads to the conclusion that the requirements are best met by the method of exact description of partial tasks in the working process including its critical features and their subsequent assignment to workers according to the degree of compliance with their skills by means of combinatorics. This method thus also enables the management of worker development by assigning appropriate tasks.
\end{abstract}

Keywords: HR; human resources; Industry 4.0; industrial revolution; digitalization; digital transformation; skill management; matrix analysis

\section{Introduction}

With the onset of the Fourth Industrial Revolution, generally known as Industry 4.0, there are new requirements which literally force production companies to reconsider their current approaches to processes so that they are able to survive in the environment of ever-increasing competition whose success is based on the automation tools, AI or marketing using social networks. New challenges must respect both production and supporting processes. In the area of human resources management, these changes are reflected mainly in the change of the approach to staff development. In the times when the unemployment rate is extremely low, the labour market does not offer enough qualified workers. Companies thus need to obtain them by training low qualified or unqualified human capital using various growth and training programmes. In the last three years, there has been lack of even unqualified workers. They are often replaced by labour force from economically less developed countries which usually have high unemployment rate.

The focus of HR managers' efforts to improve the qualification of human resources must thus dynamically shift given the current situation in the human capital market. Today we can thus see a shift from recruiting experts to working with human capital. Companies are aware of the fact that their potential success is generated mainly by appropriately allocated and qualified staff; there is thus a literal renaissance of skills monitoring, the so-called talent management. The driver of these transformation processes is represented by two main aspects in the long run - demographic and technological [1]. The demographic aspect refers mainly to the increasing average life expectancy. Combined with ever-increasing onset of automation technologies, it will bring an increase in unqualified labour force in the labour market. In the USA as the world's strongest economy, unqualified labour force accounts for $47 \%$ of all working people. About $60 \%$ of working Americans perform tasks of which up to $30 \%$ are subject to automation even now [1]. It is thus clear that organizations will have to succeed in monitoring, management and development of employee skills. It must be able to allocate a sufficient number of qualified workers in management positions and fill key medium-skilled positions with its own workers on the basis of their transformation from less qualified positions, while unqualified labour force will be gradually reduced as a result of the Fourth Industrial Revolution.

A question thus arises of how to cope with this emerging pressure and work effectively with available human capital, how to monitor the skills and competencies of employees, measure and develop them, and properly interpret the effects of growth or decline in skills. The specific benefit of this paper is the effort to find a comprehensive system that would facilitate and enable the mapping and development of employee skills using modern tools of database systems.

\footnotetext{
*Corresponding author: 27843@mail.vstecb.cz
} 


\section{Literary research}

The 21st century is a period of innovative technologies and digitization. The transformation of HRM by means of digital tools puts an external pressure on changes in human behaviour, skills and competencies in organizations [2]. Technological development enables streamlining of production processes and brings changes in human labour, which might threaten employee wellbeing and challenge their existing skills and knowledge [3]. Claus [1] addressed the need for introducing talent management due to the unfavourable development in the human capital market. Using the application of scientific knowledge from other scientific disciplines closely related to HR, the author identified the need for introducing talent management in order to become competitive in the context of the fourth industrial revolution. Mothe and Nguyen-Thi [4] analysed the relationship of age diversity of employees and the degree of technological innovations in a company. On the basis of in-depth interviews with top managers, Whysall, Owtram and Brittain [5] conclude that the fast pace of technological changes created a gap between employee skills and dynamically changing requirements for their roles. This is confirmed by Dahlbom, Siikanen, Sajasalo and Jervenpää [6], who use qualitative interviews to describe the need for interconnecting data analysts and HR specialists, who usually do not have analytical skills. The application of HRA (Human resources analysis) shall also have a positive impact on reducing the subjectivity of evaluating employees, which may result in increased willingness to optimize work performance, as confirmed by Sharma, Sharma [7] using the analysis of available professional literature in the field of HR and HRA. Zhou, Liu, Chang and Wang [8] use a questionnaire survey implemented on a sample of 211 (mostly) manufacturing companies operating in China as the world's largest labour market to describe the relationship between economic advance and digitization of HRM (Human resources management) and the improving performance of the company. The analysis of the obtained data indicates the existence of a positive correlation between a company's performance and advanced digitized HRM system. Agarwal and Maurya [9] examine the relationship of quality and the power of corporate brand the way it is perceived by public in dependence on the degree of development of talent management in the organization. Using a questionnaire survey and subsequent regression and correlation analysis, they confirm the existence of a strong dependence between the degree of development of talent management and a positive perception of the organization by the public. Jones, Hutcheson and Camba [10] argue that the current Covid-19 pandemic can positively contribute to the development and digitization of systems (not only) in the field of HR. Companies were often forced to start investing in the digitization of their processes, mainly because of work from home.

It follows from the above findings that in the context of the Fourth Industrial Revolution, the world calls for the integration of HRA, talent management, and skills management in the everyday activities of organizations. However, the integration has been addressed in few publications only. Nunes, Pinto and Sousa [11,12] propose a development framework for employee technical skills by applying a structured training system. The effectiveness of the training system is then verified by mapping the OEE (Overall equipment effectiveness) indicator. Nunes, Pinto and Sousa [11,12] represent the method of improving competencies and skills of employees in production by means of continuous improvement of production process quality. By applying the method of QC Story (The process of control and improving the quality in production), subsequent analysis of key indicators, and questionnaire survey, they conclude that the focus on the process of quality improvement leads to the improvement of key process indicators as well as to the improvement of qualification, i.e. employee skills and knowledge in the long run. Anh and Lee [13] propose the application of the knowledge and skills of current employees as a model profile of a suitable applicant for a job. By mapping and subsequent analysis of excellent employees' skills provides a certain model profile of an ideal potential job seeker. For creating the model and the compliance verification, analytical AI tools are used. It uses specific knowledge for the recruitment of employees, whose integration into the work process will be seamless; however, no future development of such employees is not provided by the system. Stadnicka, Arkhipov, Battaïa and Chandima [14] apply the model of knowledge and skills in the optimization of aircraft maintenance process as a critical process in terms of the quality and safety. By analysing the process steps in repairing a transport aircraft and subsequent assigning the necessary specific skills and knowledge from the field of technology and safety, they provide a comprehensive overview of requirements for a worker suitable for performing the specific critical task. For assigning the task to a specific employee, the so-called Hall's marriage theorem is used, i.e. combinatorial methods analysing the compliance of the requirements with the skills of a given worker. Kataoka et al. [15] focus on the application of skills management as a tool to improve the required skills of students taking their practical training. The application of the model is implemented in three steps. First, the expected skills are defined across all activities of the production process. Second, the verification methods and evaluation criteria are selected. The last step is the implementation of the model in the syllabus of specific training. The application of the training framework has a form of the so-called experiential learning. The course is repeated cyclically and its outputs are analysed until the individual reaches the required level of skills.

The available publications mostly reflect a specific, narrow field of the applicability of the system of mapping and development of employee skills. A comprehensive system applicable across processes and industries is not described.

\section{Methodology}

By studying available professional publications, we may conclude that there is a global need for the monitoring of employee skills and knowledge for the purpose of their management and development mainly in the case of low-skilled 
or unskilled positions. A matrix analysis is used to compare four selected systems of employee skills monitoring and their applicability for the manufacturing company. The selection criteria reflect the needs of the coming fourth industrial revolution and digital age. For the purpose of analysing available professional publication, the following research question was formulated: Which of the available methods best meets the requirements of the coming fourth industrial revolution?

The input alternatives for the evaluation using the matrix analysis are the systems by Nunes, Pinto and Sousa [11,12], i.e. the application of the training framework and subsequent verification of the effects on the production process by means of monitoring the OEE indicator, the systems by Stadnicka, Arkhipov, Battaïa and Chandima [14], i.e. the analysis of the partial process tasks and subsequent assigning of a suitable worker using the Hall's marriage theorem, the method used by Ahn, Lee [13], i.e. the creation of an ideal job seeker's profile by analysing the skills of excellent workers, and method used by Nunes, Pinto and Sousa [11,12], i.e. employee development by means of the application of QC story method - continuous improvement of the production process quality.

Available alternatives are compared in the following criteria:

a) multidisciplinary applicability

- the method is applicable across all production and non-production processes regardless of the field the organization operates in

b) forced costs

- implementation of the method does not incur any costs; mainly the acquisition costs of software in which the method is implemented

c) need for IT experts from practice (implementation and maintenance autonomy)

- implementation does not require hiring IT experts, the entity is able to manage the implementation without any help of IT experts $\rightarrow$ development of IT skills

d) permanent sustainability

- the methodology is repeatedly applicable; it reflects the current development in the field of IT as well as the automation of the production processes in the context of Industry 4.0

e) focus on personal growth

- the method is used for the development and management of an individual's talent; it is applicable as motivation connected with the corporate remuneration system

f) comprehensibility at all organizational levels

- all employees in the organization understand the basis of the methodology, understand what is being monitored, and for which purpose

g) reduced evaluation subjectivity

- the methodology is transparent enough so that it does not diminish employee trust and personal engagement

The strength of the relationship between the variant and evaluation criterion is presented at four levels (strong, medium, weak, and no relationship), where no relationship represents the minimal or no match of the solution variant with the required criterion, while strong relationship refers to the absolute match. In the case of the evaluation criteria forced costs and need for IT experts from practice, the logic of assigning characteristic strengths of the features is logically reversed, i.e. the higher the need, the weaker the relationship and the lower degree of match with the required evaluation criterion. The strength of the relationship is then converted into a point scale $(0,5,10,15$ points $)$. Summing of the points provides an accurate selection of the most suitable method showing the highest degree of match across the evaluation criteria.

\section{Results}

Table 1 shows a matrix analysis, i.e. the expression of the strength of the relationship between the selection criterion on the horizontal axis and the solution variant on the vertical axis. The strength of the relationship is then assigned based on the knowledge obtained from the specific professional papers on the scale of no relationship - strong relationship. 
Table 1. Matrix analysis of available solutions

\begin{tabular}{|l|l|}
\hline$\bigcirc$ & no relationship \\
\hline$\triangle$ & weak relationship \\
\hline (O) & medium relationship \\
\hline (O) & strong relationship \\
\hline
\end{tabular}

\begin{tabular}{|c|c|c|c|c|c|c|}
\hline 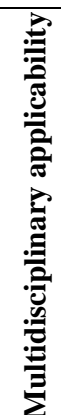 & 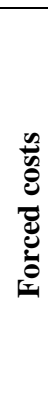 & 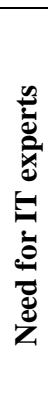 & 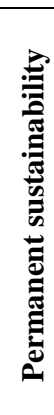 & 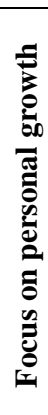 & 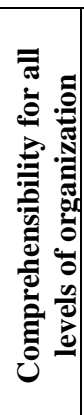 & 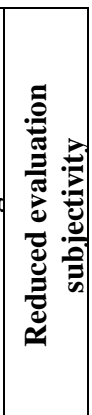 \\
\hline & & & & & & \\
\hline (0) & & (O) & & (O) & (0) & \\
\hline & & & & & (0) & \\
\hline & & & (0) & & & \\
\hline
\end{tabular}

Source: Authors.

Table 2 shows the exact numerical expression based on the strength of the relationship (0-15 points). The last column of the table contains the overall sum of the points. The available methods under review show the compliance of the results of the criterion permanent sustainability, where three of them show a strong relationship; on the contrary, there is weak or no relationship in the case of the criterion reduced evaluation subjectivity. It can thus be concluded that the methods are applicable in the context of the fourth industrial revolution; however, it is necessary to develop the transparency leading to the reduced subjectivity of the evaluation of the employee, especially if the evaluation is connected with the remuneration system. The criterion of forced costs also shows match across all implementation schemes. This finding can be interpreted as a need for high input costs of the implementation of the method, mainly due to the dynamically changing requirements combined with the lack of HR staff skills, as confirmed by Dahlbom, Jervenpää, Sajasalo and Siikanen [6]. Particularly high implementation costs can be seen in the case of the implementation scheme No. 3 by Lee and Anh, which compares the candidate's compliance with the profile of an ideal worker using AI analytical tools; autonomous implementation and administration from the side of the organization cannot be assumed there. On the contrary, the second implementation scheme shows the best results in the comparison with the requirements of the fourth industrial revolution, as at least medium strength of the relationship is achieved in the case of five out of seven criteria.

Table 2. Numerical expression of the strength of the relationship, criterion - variant

\begin{tabular}{|c|c|c|c|c|c|c|c|c|c|c|c|}
\hline $\begin{array}{c}\text { Solution } \\
\text { No. }\end{array}$ & Author & Published & Method & 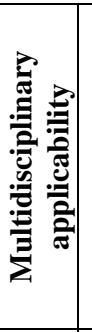 & 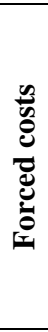 & 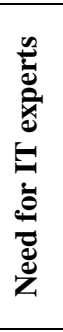 & 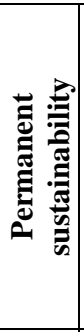 & 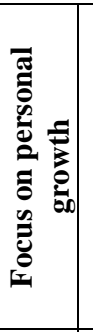 & 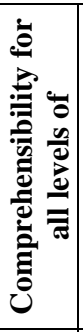 & 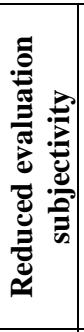 & $\sum$ \\
\hline 1 & Pinto, Nunes, Sousa & 2020 & $\begin{array}{l}\text { application of training } \\
\text { framework }+ \text { verification by } \\
\text { means of OEE indicator }\end{array}$ & 5 & 5 & 10 & 15 & 0 & 5 & 0 & 40 \\
\hline 2 & $\begin{array}{l}\text { Stadnicka, } \\
\text { Arkhipov, Battaïa, } \\
\text { Ratnayake }\end{array}$ & 2017 & $\begin{array}{l}\text { analysis of process and its } \\
\text { critical features - assigning } \\
\text { operator using Hall's marriage } \\
\text { theorem }\end{array}$ & 15 & 5 & 10 & 15 & 10 & 15 & 5 & 75 \\
\hline 3 & Lee, Anh & 2020 & $\begin{array}{l}\text { creation of an ideal job } \\
\text { seeker's profile }+ \text { verification } \\
\text { of the compliance using AI } \\
\text { analytical tools }\end{array}$ & 10 & 0 & 0 & 5 & 15 & 10 & 5 & 45 \\
\hline 4 & $\begin{array}{l}\text { Souza, Nunes, } \\
\text { Sousa }\end{array}$ & 2018 & $\begin{array}{l}\text { monitoring of the } \\
\text { development using the QC } \\
\text { story method }\end{array}$ & 5 & 5 & 10 & 15 & 0 & 5 & 0 & 40 \\
\hline
\end{tabular}

Source: Authors. 


\section{Discussion}

The total sum of the points of expressed strength of the relationship between the criterion and implementation variant indicates the most suitable method, which turns out to be the method No. 2, system developed by Stadnicka, Arkhipov, Battaïa and Chandima [14] based on the analysis of individual partial tasks and their subsequent assigning to individual workers according to their skills and knowledge, as it best meets the requirements arising from the coming fourth industrial revolution. The system is applicable across industries and processes. In its implementation and management, the participation of IT experts is not necessary. The system is sustainable and sufficiently flexible, focusing on the development of individuals and its transparency enables the reduction of the evaluation subjectivity. Also, the system of assigning specific tasks to employees on the basis of a detailed analysis of the task requirements and employee skills appears to be the most suitable available solution. The system enables the management of employee development by means of assigning tasks of various degrees of difficulty and critical features. The weakness is the overall transparency leading to increasing trust. This is represented by the need to apply the combinatorial method, the so-called Hall's marriage theorem, to exactly assign a task to a suitable worker.

The imaginary last position is occupied by the systems used by Nunes, Pinto and Sousa [11,12]. These systems show the lowest degree of compliance especially with the criteria of focus on individual and the overall transparency of the system. Development is mapped by means of monitoring the indicators which are not directly related to the development of employees. Employee development is a secondary effect to the improvement of the production in the area of production quality or increasing the usability of machinery. The system is thus not fully transparent for employees and a question arises of "What is the share of individual employees in improving the indicators?" Therefore, this is not a suitable method of skills mapping with the potential connection to the organization remuneration system.

The system developed by Anh, Lee [13] shows the highest possible degree of compliance for the criterion "focus on individuals", since the system creates the profile of an ideal employee on the basis of actual knowledge from practice and subsequently seeks for a suitable candidate with the highest degree of compliance with the profile. The main disadvantages can be seen in a very complex creation of the profile, which requires the participation of the IT experts from practice as well as high additional acquisition costs of software. Another disadvantage is its single-purpose use only in the stage of recruitment.

\section{Conclusion}

The objective of the paper was to determine how to meet the requirements of the HR system transformation, especially in the field of employee development in the context of the fourth industrial revolution and coming digital transformation. For this purpose, available professional texts concerning the given issue were analysed. From these texts, four specific implementation criteria were selected, which were put into the context of seven criteria meeting the basic ideas of the fourth industrial revolution and digital transformation, namely multidisciplinary applicability, minimal costs, reduction of the need for experts, permanent sustainability of the system, focus on the growth of individuals, transparency of the whole system, and efforts for the reduction of subjectivity in evaluating employees. The strength of the relationship between the implementation scheme and evaluation criterion was divided into four levels (strong, medium, weak, and no relationship), which were assigned relevant number of points on the scale of 0-15. Subsequently, matrix analysis of the strength of the relationship between the requirements arising from the fourth industrial revolution in HR and available solutions was performed. By summing the points assigned to the strength of the relationships, it can be concluded that the requirements of the fourth industrial revolution are best reflected and met by the implementation scheme No. 2 , i.e. the authors Stadnicka, Arkhipov, Battaïa and Chandima [14], specifically, the system based on the exact knowledge of partial steps of a task including the definition of critical features and the subsequent assignment of the tasks to individual operators using the combinatorial method, the so-called Hall's marriage theorem. However, the method of assigning a task to a specific employee may pose pitfalls in the case of its implementation in a small company due to its complexity and worse transparency of the system. After the exact analysis of partial steps of the working process, a question arises of how to find a suitable worker in a form understandable to the employees themselves. Therefore, there is some space for further research aimed at improving the transparency of the system across the organizational levels of the company in order to increase the trustworthiness of the system.

\section{References}

1. L. Claus. HR disruption - Time already to reinvent talent management. BRQ Business Research Quarterly. 22(3), 207-215 (2019)

2. A. Sengupta, S. Lalwani, S. Goswami, P. Srivastava. Reinventing HR functions with SMAC technologies - an exploratory study. Materials Today: Proceedings. 46(1) (2020)

3. A. Reiman, J. R. L. Kaivo-Oja, E.-P. Takala, T. Lauraeus, E. Parviainen. Human factors and ergonomics in manufacturing in the industry 4.0 context - A scoping review. Technology in Society. 65 (2021) 
4. C. Mothe, T. U. Nguyen-Thi. Does age diversity boost technological innovation? Exploring the moderating role of HR practices. European Management Journal. (2021)

5. Z. Whysall, M.Owtram, S. Brittain. The new talent management challenges of Industry 4.0. Journal of Management Development. 38(3) (2019)

6. P. Dahlbom, M. Jarvenpää, P. Sajasalo, N. Siikanen. Big data and HR analytics in the digital era. Baltic Journal of Management. 15(1) (2019)

7. A. Sharma, T. Sharma. HR analytics and performance appraisal system: A conceptual framework for employee performance improvement. Management Research Review. 40(6), 684-697 (2017)

8. M. Agarwal, K. K. Maurya. Organisational talent management and perceived employer branding. International Journal of Organizational Analysis. 26(2) (2018)

9. Y. Zhou, X. Chang, G. Liu, L. Wang. The impact of HRM digitalization on firm performance: investigating threeway interactions. Asia Pacific Journal of Human Resources. 59(1), 20-43 (2021)

10. J. D. Camba, S. Hutcheson, M. D. Jones, S. Hutcheson. Past, present, and future barriers to digital transformation in manufacturing: A review. Journal of Manufacturing Systems. 60(9) (2021)

11. R. Sousa, S. Souza, E. Nunes. Developing organisational learning through QC story. Total Quality Management \& Business Excellence. 31(13-14), 1565-1587 (2020)

12. E. M. P. Nunes, L. Pinto, S. D. Sousa. A framework to improve training and development of workers' technical skills: effects on operational performance during company relocation. Procedia Manufacturing. 51(6), 1806-1813 (2020)

13. CH. Ahn, D. Lee. Industrial human resource management optimization based on skills and characteristics. Computers \& Industrial Engineering. 144 (2020)

14. D. Stadnicka, D. I. Arkhipov, O. Battaïa, R. M. Chandima. Skills management in the optimization of aircraft maintenance processes. IFAC World Congres 2017. 50(1), 6912-6917 (2017)

15. T. Kataoka, K. Morikawa, K. Takahashi. Strategic Human Resource Management Simulation Considering Work Elements, Skills, Learning and Forgetting. 25th International Conference on Production Research Manufacturing Innovation: Cyber Physical Manufacturing. 39, 633-1640. (2019) 